\title{
Single Stage Oncologic Resection and Reconstruction: A Step toward Development of Sarcoma Service in Resource Constrained Country ${ }^{*}$
}

\author{
Haroon ur Rashid ${ }^{1}$, Kashif Abbas ${ }^{2 \#}$, Masood Umer ${ }^{1}$ \\ ${ }^{1}$ The Aga Khan University Hospital, Karachi, Pakistan; ${ }^{2}$ Islam Medical and Dental College, Sialkot, Pakistan. \\ Email: "kashah_pk@yahoo.com \\ Received February 28 ${ }^{\text {th }}, 2013$; revised March 27 ${ }^{\text {th }}$ 2013; accepted April $5^{\text {th }}, 2013$ \\ Copyright (C) 2013 Haroon ur Rashid et al. This is an open access article distributed under the Creative Commons Attribution License, \\ which permits unrestricted use, distribution, and reproduction in any medium, provided the original work is properly cited.
}

\begin{abstract}
Tumor free-margin surgical resection remains the single most important treatment in the curative therapy of musculoskeletal tumor of limbs. Refinements in surgical techniques have led to increased function preservation and limb salvage. Patients and Methods: The records of patients $(n=24)$ who underwent microsurgical soft tissue reconstruction subsequent to resection of limb tumour during the period 2006 to 2011 were reviewed. Primary outcome i.e. uptake of the flap was evaluated. Perioperative morbidities were also noted including donor as well as recipient site complications. Assessment of Functional outcome (Musculoskeletal Tumor Society score, MSTS) local recurrence, free survival, and disease-specific survival was also made. Results: Twenty four patients (age range: 7 - 72 years) who have undergone tumor resection followed by flap coverage were identified. Lower limb reconstruction outnumbered upper limb by 6:1. Complications included, one complete failure of free vascularized iliac crest flap done for reconstruction of a heel defect. One of the patients had secondary hemorrhage 10 days after surgery. Another patient with internal hemipelvectomy for Ewing's sarcoma had a dura puncture during resection of sacrum. Partial epidermal necrosis was evident in four cases. Eighty three percent of the patients remained alive $(n=20), 19$ of whom currently have no evidence of disease (NED) Disease recurrence was noted in three patients. Overall MSTS score was 73.5\%. Conclusion: The microsurgical repair of defects is a reliable option that, though not free of complications, is necessary in selected cases. The procedure enables both adequate oncosurgical resection and function preservation.
\end{abstract}

Keywords: Musculoskeletal Tumor; Reconstruction; Flap

\section{Introduction}

Survival rates after limb salvage surgery have improved greatly over the past 20 years, primarily because of new techniques in soft tissue reconstruction. The required surgical margin of $2-3 \mathrm{~cm}$ of tumor free tissue frequently causes large soft tissue defects. Local or free flaps are often required to achieve tension free wound closure or to reconstruct tissue defects.

The basic principles in soft tissue vascular anatomy and bony reconstruction have long been established. In the last two decades treatment of soft tissue defects has become commonly available and reliable. Today, using a combination of surgery and radiotherapy, better functional results are achieved with equal rates of local con-

*Disclosure: No benefits in any form have been received or will be received related directly or indirectly to the subject of this article.

${ }^{\#}$ Corresponding author. trol.

A viable yet painful, stiff or insensate limb hardly serves the patient; the instinctive desire on both the patient and physicians' part to save a limb at all costs must be tempered by the expected long-term functional result. The most heroic and beautifully performed vascular and bony reconstructions are wasted without concomitant coverage of these repairs [1,2].

Modern treatment consists of multidisciplinary team approach; orthopedic oncologist resects the tumor and reconstructs the skeletal defect which is followed by the second team doing the soft tissue reconstruction work [3].

In advanced cases and delayed presentations, limb salvage is impossible, and sometimes amputation is unavoidable [4]. For very proximal shoulder or pelvic girdle resections, soft tissue reconstruction may not be pos- 
sible without a rotational or free flap [5].

The aim of the study is to evaluate the results of our cases of microvascular reconstruction done for extensive bone and soft tissue defects after tumor resection.

\section{Materials and Methods}

This is a retrospective review of twenty four patients who underwent reconstruction of oncologic defects by a single team of two surgeons at a single institution from 2006-2011. All patients with tumors of extremities requiring soft tissue reconstruction for wound closure were included. We excluded cases that required split or full thickness skin grafting as a sole means of wound coverage. Medical record number are retrieved through surgical team database and demographics and further details were reviewed through confidential files and hospital based software called Patient Care Inquiry (PCI), containing patient records of hospital visits. Primary outcome i.e. uptakes of the flap were evaluated. Perioperative morbidities were also noted including donor as well as recipient site complications. The Musculoskeletal tumor society (MSTS) score is a clinical scored system assessing pain, function, and emotional acceptance in patients for upper and lower extremities. Patients with lower extremity reconstructions were also evaluated with regard to walking ability, gait, and the use of walking aids. Patients with upper extremity reconstructions were evaluated for manual dexterity, hand positioning, and lifting ability.

\section{Surgical Team Protocol}

Our surgery team comprises of two surgeons each specialized in tumor surgery and soft tissue reconstruction.

All surgeries were done under general anesthesia. Preoperative dose of Tranexamic acid $1 \mathrm{~g}$ (to reduce post-operative blood loss) and cefazolin $1 \mathrm{gm}$ is a routine at the time of induction. Most of the surgeries below mid-thigh level were done with tourniquet. Reconstruction followed immediately after tumor resection and was done by the second surgeon with new sets of instruments. Microsurgical aids were used where required. In three cases of vascularized fibula, the procedure (tumor resection and reconstruction) was started simultaneously with different set of instruments and scrubbed personnel to minimize overall surgical duration.

Postoperative flap monitoring was done on hourly basis for initial 12 hours followed by 4 hourly monitoring. Initial dressing change is done after 3 days and patients are usually discharged after 5 days. Outpatient follow up is weekly for first 3 weeks followed by monthly visit for next 3 months. Patients are then followed up each quarter for next 5 years. Patient living in remote cities were followed on phone and mail.

\section{Results}

Twenty four patients were identified who have undergone tumor resection followed by flap coverage. There were thirteen males (54\%) and 11 females (46\%). Mean age was 29 years ( 7 - 72 years), reflecting mix population of diverse age group. Pathologic diagnosis of osteosarcoma was present in 10 patients (42\%) followed by 4 patients (17\%) with soft tissue sarcoma, 3 patients (12\%) with Ewing sarcoma and squamous cell carcinoma of extremities each, 2 (8\%) with malignant melanoma of extremities, one (4\%) each of chondrosarcoma and giant cell tumor. Tissue diagnosis was available in all patients preoperatively. Lower limb reconstruction (88\%) outnumbered upper limb by 6:1. Eighty three percent of the patients remained alive $(n=20), 19$ of whom currently have no evidence of disease (NED) Disease recurrence was noted in three patients (13\%), two patients underwent wide margin resection for malignant melanoma, both of which had recurrence within 2 years. A more radical procedure in the form of forearm amputation was done in one and the second patient refused any further intervention and was subsequently lost to follow. Another patient with pleomorphic sarcoma of the proximal tibia also had recurrence of disease, remote from the site of surgery within 6 months; he also lost to follow subsequently. One patient died of metastatic disease and another patient with ewing sarcoma of pelvis died during the course of her treatment after 12 months of surgery (Table 1).

Complications included, one complete failure of free vascularized iliac crest flap done for reconstruction of a heel defect. This was subsequently managed with vacuum dressing and secondary wound closure. Another patient had secondary hemorrhage 10 days after surgery. Reconstruction involved in this case was coverage of post external hemipelvectomy wound defect with free flap harvested from amputated limb and anastomosed with external iliac vessels. Patient was rushed to operative room due to expanding hematoma and drop in hemoglobin. Intraoperative finding were consistent with generalized ooze, thus wound was closed over drains; the flap survived without any further complication. Another patient with internal hemipelvectomy for Ewing's sarcoma had a dura puncture during resection of sacrum for which a rectus abdominus flap and lumbar drain was placed, postoperative recovery was uneventful and the drain was removed after 5 days (Table 2).

Partial epidermal necrosis was evident in four cases whereas wound infection was observed in three patients.

\section{Discussion}

The goal of limb salvage surgery is to obtain safe surgical margins, preserving length and function. Simultane- 
Table 1. Patient demographics.

\begin{tabular}{|c|c|c|c|c|c|c|c|c|c|c|c|c|}
\hline & Gender & $\begin{array}{l}\text { Age } \\
\text { (years) }\end{array}$ & Site & Biopsy & Surgery & Flap & $\begin{array}{l}\text { Follow } \\
\text { up } \\
\text { (months) }\end{array}$ & $\begin{array}{c}\text { Status at } \\
\text { last follow } \\
\text { up }\end{array}$ & Complication & Recurrence & $\begin{array}{l}\text { Flap } \\
\text { dimensions }\end{array}$ & $\begin{array}{l}\text { MSTS } \\
\text { score }\end{array}$ \\
\hline 1 & Male & 65 & Right foot & $\begin{array}{l}\text { Malignant } \\
\text { melanoma }\end{array}$ & $\begin{array}{c}\text { Wide margin } \\
\text { excision + lymph } \\
\text { node dissection }\end{array}$ & $\begin{array}{c}\text { Medial } \\
\text { plantar } \\
\text { artery flap }\end{array}$ & $\begin{array}{c}30 \\
\text { months }\end{array}$ & $\begin{array}{c}\text { Recurrence: } \\
24 \text { months }\end{array}$ & Nil & $\begin{array}{c}\text { Yes } \\
\text { (nodule } \\
\text { after } 2 \\
\text { years- } \\
\text { biopsy } \\
\text { proven) }\end{array}$ & $8 \times 5 \mathrm{~cm}$ & 72 \\
\hline 2 & Female & 64 & Right wrist & $\begin{array}{l}\text { Malignant } \\
\text { melanoma }\end{array}$ & $\begin{array}{c}\text { Wide margin } \\
\text { excision + lymph } \\
\text { node dissection }\end{array}$ & $\begin{array}{l}\text { Posterior } \\
\text { interroseous } \\
\text { artery flap }\end{array}$ & $\begin{array}{c}36 \\
\text { months }\end{array}$ & $\begin{array}{c}\text { Recurrence: } \\
18 \text { months }\end{array}$ & Nil & $\begin{array}{l}\text { Yes-tow } \\
\text { years after } \\
\text { first } \\
\text { surgery }\end{array}$ & $10 \times 6 \mathrm{~cm}$ & 60 \\
\hline 3 & Male & 37 & $\begin{array}{c}\text { Left } \\
\text { scapula } \\
\text { metastatic } \\
\text { from } \\
\text { primary } \\
\text { larynx }\end{array}$ & $\begin{array}{l}\text { Squamous cell } \\
\text { carcinoma }\end{array}$ & $\begin{array}{l}\text { Scapulectomy } \\
\text { with wide } \\
\text { margins excision }\end{array}$ & $\begin{array}{l}\text { Latissimus } \\
\text { dorsi flap + } \\
\text { STSG }\end{array}$ & $\begin{array}{c}38 \\
\text { months }\end{array}$ & Died & $\begin{array}{l}\text { Partial } \\
\text { epidermal } \\
\text { necrosis }\end{array}$ & No & $18 \times 10 \mathrm{~cm}$ & 65 \\
\hline 4 & Male & 28 & Distal leg & $\begin{array}{l}\text { Malignant } \\
\text { fibrous } \\
\text { histiocytoma }\end{array}$ & $\begin{array}{l}\text { Wide margin } \\
\text { excision and } \\
\text { tendo Achilles } \\
\text { reconstruction }\end{array}$ & $\begin{array}{l}\text { Posterior tibial } \\
\text { island flap }\end{array}$ & $\begin{array}{c}30 \\
\text { months }\end{array}$ & NED & Nil & Nil & $12 \times 6 \mathrm{~cm}$ & 78 \\
\hline 5 & Male & 28 & Left knee & $\begin{array}{l}\text { Fibrosarcoma on } \\
\text { the background } \\
\text { of dermatomfi- } \\
\text { brosarcoma } \\
\text { protuberans }\end{array}$ & $\begin{array}{l}\text { Wide margin } \\
\text { excision }\end{array}$ & $\begin{array}{l}\text { Sural artery } \\
\text { flap }\end{array}$ & $\begin{array}{c}38 \\
\text { months }\end{array}$ & NED & Nil & Nil & $10 \times 5 \mathrm{~cm}$ & 82 \\
\hline 6 & Male & 44 & Right heel & $\begin{array}{l}\text { Squamous cell } \\
\text { carcinoma }\end{array}$ & $\begin{array}{c}\text { Wide margin } \\
\text { excision + tendo } \\
\text { Achilles } \\
\text { reconstruction }\end{array}$ & $\begin{array}{l}\text { Supramalleolar } \\
\text { flap }\end{array}$ & $\begin{array}{c}20 \\
\text { months }\end{array}$ & NED & Nil & Nil & $8 \times 4 \mathrm{~cm}$ & 86 \\
\hline 7 & Male & 40 & $\begin{array}{l}\text { Right neck } \\
\text { of femur }\end{array}$ & $\begin{array}{l}\text { Osteogenic } \\
\text { sarcoma }\end{array}$ & $\begin{array}{l}\text { Hindquarter } \\
\text { amputation }\end{array}$ & Fillet flap & $\begin{array}{c}34 \\
\text { months }\end{array}$ & NED & $\begin{array}{l}\text { Secondary } \\
\text { hemorrhage }\end{array}$ & Nil & $20 \times 14 \mathrm{~cm}$ & 68 \\
\hline 8 & Female & 13 & $\begin{array}{l}\text { Left } \\
\text { proximal } \\
\text { tibia }\end{array}$ & $\begin{array}{l}\text { Osteogenic } \\
\text { sarcoma }\end{array}$ & $\begin{array}{l}\text { Wide margin } \\
\text { excision }\end{array}$ & $\begin{array}{l}\text { Sural artery } \\
\text { flap }\end{array}$ & $\begin{array}{c}24 \\
\text { months }\end{array}$ & NED & Nil & Nil & $9 \times 5 \mathrm{~cm}$ & 84 \\
\hline 9 & Female & 19 & $\begin{array}{l}\text { Right } \\
\text { proximal } \\
\text { tibia }\end{array}$ & $\begin{array}{l}\text { Osteogenic } \\
\text { sarcoma }\end{array}$ & $\begin{array}{l}\text { Wide margin } \\
\text { excision }\end{array}$ & $\begin{array}{c}\text { Tibialization + } \\
\text { sural artery flap }\end{array}$ & $\begin{array}{c}24 \\
\text { months }\end{array}$ & NED & Nil & Nil & $10 \times 5 \mathrm{~cm}$ & 86 \\
\hline 10 & Male & 37 & $\begin{array}{l}\text { Left } \\
\text { popliteal } \\
\text { mass }\end{array}$ & $\begin{array}{l}\text { Pleomorphic } \\
\text { sarcoma }\end{array}$ & $\begin{array}{l}\text { Wide margin } \\
\text { excision }\end{array}$ & $\begin{array}{c}\text { Gastrocnemius } \\
\text { flap + STSG }\end{array}$ & 6 months & $\begin{array}{l}\text { Recurrence: } \\
\text { lost to } \\
\text { follow up }\end{array}$ & Nil & $\begin{array}{l}\text { Recurrence } \\
\text { mid thigh } \\
\text { region } \\
\text { anteriorly }\end{array}$ & $8 \times 5 \mathrm{~cm}$ & 78 \\
\hline 11 & Male & 58 & $\begin{array}{l}\text { Left } \\
\text { forearm } \\
\text { mass }\end{array}$ & $\begin{array}{c}\text { Pleomorphic } \\
\text { leiomyosarcoma }\end{array}$ & $\begin{array}{l}\text { Wide margin } \\
\text { excision }\end{array}$ & $\begin{array}{c}\text { Free } \\
\text { osteocutanous } \\
\text { fibular flap }\end{array}$ & $\begin{array}{c}40 \\
\text { months }\end{array}$ & NED & Nil & Nil & $14 \times 6 \mathrm{~cm}$ & 80 \\
\hline 12 & Male & 44 & $\begin{array}{l}\text { Right } \\
\text { shoulder } \\
\text { mass }\end{array}$ & Chondrosarcoma & $\begin{array}{l}\text { Forequarter } \\
\text { amputation }\end{array}$ & Fillet flap & $\begin{array}{c}36 \\
\text { months }\end{array}$ & NED & $\begin{array}{l}\text { Wound } \\
\text { infection }\end{array}$ & Nil & $14 \times 8 \mathrm{~cm}$ & 70 \\
\hline 13 & Female & 10 & $\begin{array}{l}\text { Right ilium } \\
\text { mass }\end{array}$ & $\begin{array}{c}\text { Ewing } \\
\text { sarcoma }\end{array}$ & $\begin{array}{c}\text { Internal } \\
\text { hemipelvectomy }\end{array}$ & $\begin{array}{l}\text { Rectus } \\
\text { abdominis } \\
\text { muscle flap }\end{array}$ & $\begin{array}{c}12 \\
\text { months }\end{array}$ & DIED & $\begin{array}{l}\text { Epidermal } \\
\text { necrosis }\end{array}$ & Nil & $8 \times 6 \mathrm{~cm}$ & 65 \\
\hline
\end{tabular}




\begin{tabular}{|c|c|c|c|c|c|c|c|c|c|c|c|}
\hline 14 Female & 20 & $\begin{array}{c}\text { Calcaneum } \\
\text { mass }\end{array}$ & $\begin{array}{l}\text { Osteogenic } \\
\text { sarcoma }\end{array}$ & WME & $\begin{array}{l}\text { Free iliac crest } \\
\text { flap }\end{array}$ & 32 & NED & Flap failure & Nil & $6 \times 4 \mathrm{~cm}$ & 75 \\
\hline 15 Female & 20 & $\begin{array}{l}\text { Iliac crest } \\
\text { mass }\end{array}$ & $\begin{array}{l}\text { Ewing } \\
\text { sarcoma }\end{array}$ & $\begin{array}{c}\text { Internal } \\
\text { hemipelvectomy }\end{array}$ & $\begin{array}{c}\text { Rectus } \\
\text { abdominus flap }\end{array}$ & 6 & NED & $\begin{array}{c}\text { Epidermal } \\
\text { necrosis + } \\
\text { dura } \\
\text { puncture + } \\
\text { sciatic palsy }\end{array}$ & & $8 \times 6 \mathrm{~cm}$ & 68 \\
\hline 17 Male & 17 & $\begin{array}{l}\text { Right } \\
\text { proximal } \\
\text { tibia }\end{array}$ & Osteosarcoma & $\begin{array}{l}\text { Wide margin } \\
\text { excision }\end{array}$ & $\begin{array}{l}\text { Sural artery } \\
\text { flap }\end{array}$ & 14 & NED & $\begin{array}{l}\text { Infection/ } \\
\text { nonuncion }\end{array}$ & & $8 \times 4 \mathrm{~cm}$ & 82 \\
\hline 18 Female & 24 & $\begin{array}{l}\text { Right } \\
\text { distal } \\
\text { femur } \\
\text { mass }\end{array}$ & Giant cell tumor & $\begin{array}{l}\text { Extra articular } \\
\text { resection }\end{array}$ & $\begin{array}{l}\text { Gastrocnemius } \\
\text { flap }\end{array}$ & 24 & NED & $\begin{array}{l}\text { Flap failure/ } \\
\text { sural flap/ } \\
\text { free } \\
\text { lattissimus } \\
\text { dorsi flap }\end{array}$ & & $\begin{array}{c}6 \times 4 \mathrm{~cm} \\
\left(1^{\mathrm{st}}\right) \\
8 \times 4 \mathrm{~cm} \\
\left(2^{\text {nd }}\right) \\
14 \times 8 \mathrm{~cm} \\
\left(3^{\mathrm{rd}}\right)\end{array}$ & 62 \\
\hline 19 Male & 38 & Right tibia & $\begin{array}{l}\text { Squamous cell } \\
\text { carcinoma of } \\
\text { right leg }\end{array}$ & $\begin{array}{l}\text { Wide margin } \\
\text { excision }\end{array}$ & $\begin{array}{c}\text { Free latissimus } \\
\text { dorsi flap }\end{array}$ & 6 & NED & $\begin{array}{c}\text { STSG } \\
\text { failure/ } \\
\text { healed by } \\
\text { secondary } \\
\text { intention }\end{array}$ & & $\begin{array}{c}16 \times 10 \\
\mathrm{~cm}\end{array}$ & 78 \\
\hline 20 Female & 20 & $\begin{array}{l}\text { Left Distal } \\
\text { femur } \\
\text { Mass }\end{array}$ & Osteosarcoma & $\begin{array}{l}\text { Extraarticular } \\
\text { resection of knee } \\
\text { mass }\end{array}$ & $\begin{array}{l}\text { Gastrocnemius } \\
\text { flap/free } \\
\text { latissimus } \\
\text { dorsi flap }\end{array}$ & 20 & NED & $\begin{array}{l}\text { Initial wound } \\
\text { dehiscence }\end{array}$ & & $\begin{array}{c}7 \times 4 \mathrm{~cm} \\
\left(1^{\mathrm{st}}\right) \\
12 \times 7 \mathrm{~cm} \\
\left(2^{\text {nd }}\right)\end{array}$ & 70 \\
\hline 21 Female & 14 & $\begin{array}{c}\text { Distal } \\
\text { femur } \\
\text { mass }\end{array}$ & Osteosarcoma & $\begin{array}{l}\text { Wide margin } \\
\text { excision }\end{array}$ & $\begin{array}{l}\text { Vascularized } \\
\text { fibula }\end{array}$ & 14 & NED & & & $\begin{array}{l}\text { No } \\
\text { cutaneous } \\
\text { island }\end{array}$ & 72 \\
\hline 23 Female & 13 & $\begin{array}{l}\text { Right mid } \\
\text { femur } \\
\text { mass }\end{array}$ & Osteosarcoma & $\begin{array}{l}\text { Wide margin } \\
\text { resection }\end{array}$ & $\begin{array}{l}\text { Vascularized } \\
\text { fibula }\end{array}$ & 6 & NED & & & $\begin{array}{c}\text { No } \\
\text { cutaneous } \\
\text { island }\end{array}$ & 72 \\
\hline 24 Male & 12 & $\begin{array}{l}\text { Distal } \\
\text { femur } \\
\text { lesion }\end{array}$ & Osteosarcoma & $\begin{array}{l}\text { Wide margin } \\
\text { resection }\end{array}$ & $\begin{array}{l}\text { Vascularized } \\
\text { fibula }\end{array}$ & 10 & NED & & & $\begin{array}{l}\text { No } \\
\text { cutaneous } \\
\text { island }\end{array}$ & 68 \\
\hline
\end{tabular}

Table 2. Peripoperative complications (n = 27 flaps in 24 patients).

\begin{tabular}{cc} 
Death & 0 \\
\hline Flap failure & 3 \\
\hline Recipient site Morbidity & \\
Wound breakdown & 4 \\
Seroma/hematoma & 3 \\
Hemorrhage & 1 \\
Infection & 3 \\
Poor graft uptake & 1 \\
Dura puncture & Others \\
\hline
\end{tabular}

ous tumor resection and soft tissue reconstruction have obvious functional benefits [6].

Selection of flaps varies with location of primary disease and extent of resection. Pedicle based flaps are always preferred over free flaps. Gastrocnemius muscle flap is a salvage option for soft tissue reconstruction around knee joint and is also helpful in providing pliable tissue cushion around neurovascular structures. The medial or lateral heads of the gastrocnemius muscle can be expended with little or no deficit when walking or in normal running [7].

In our series, gastrocnemius flaps were done in two patients after extra-articular resection of knee joint in order to cover implants and prevent overlying skin breakdown. Both of these patients had epidermal necrosis and subsequent dehiscence of wound within two weeks of 
index surgery due to excessive undermining of skin flaps for tumor resection. Free myocutaneous latissimus dorsi flap was done to cover the defect in first patient and had an uneventful postoperative recovery. In the second patient, sural artery pedicle flap was done initially which failed gradually within 3 weeks; this was followed by a free latissimus dorsi flap, which proved to be the final procedure in this patient. Third gastrocnemius flap was done for coverage of wound defect in popliteal fossa to provide cushion around neurovascular structure. Wound healing was uneventful in this patient.

In a study by Liu $\mathrm{T}$ et al, group with the transposition of medial gastrocnemius muscle flap, local skin necrosis occurred in 2 (5.7\%), and prosthesis deep infection occurred in 1 (2.9\%). In the group without the transposition of medial gastrocnemius muscle flap, subctaneous hematocele, and effusion occurred in $10.0 \%$, wound infection occurred in 4 (13.3\%), 1 cured and the other 3 developed prosthesis deep infection.There was significant difference in the rate of local complications $(\mathrm{P}<0.05)$. There was significant difference in function assessment between the 2 groups $(\mathrm{P}<0.05)$. Results of patients in our study was similar, although on a low scale, both of our patient with extra articular resection and prosthetic

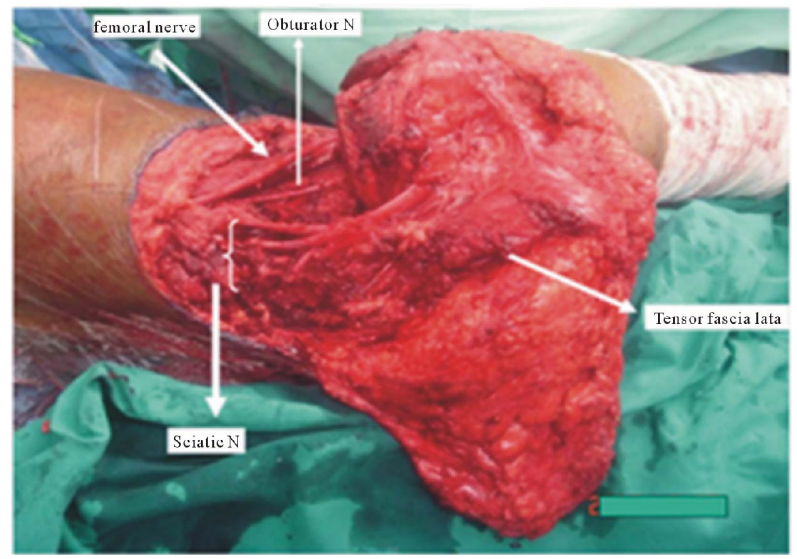

(a)

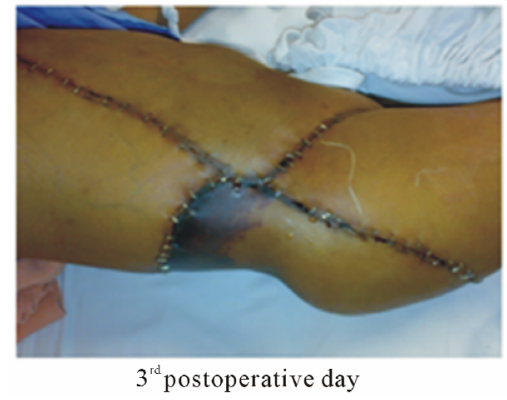

(c) reconstruction, epiderma necrosis did not result in prosthetic deep infection due to cushion provided by gastrocnemius flap [8].

For decades, rectus abdominis flap has been used to reconstruct breast defects, primarily as a pedicled flap based on superior epigastric artery. While using inferior epigastric artery as a pedicle, flap can be used to cover upper part of thigh especially in cases of hemipelvectomy where the flaps are so thinned out that wound dehiscence is likely, resulting in direct exposure of underlying neurovascular structures after tumour resection. The flap provides healthy muscle, with or without a skin paddle that can be used to replace soft tissue bulk and is easy to perform and does not require microsurgical technique $[9,10]$. In our series only muscular portion of the flap were used for soft tissue cushion. Rectus abdominis flap was done in three patients who underwent internal hemipelvectomy for pelvic tumor; one of them developed wound dehiscence following epidermal necrosis. Her wound was managed conservatively with dressings and healed with secondary intention (Figure 1).

The concept of spare part surgery is also prevalent in musculoskeletal oncology [11,12]. Reconstruction of the defects is done using amputated limbs, myocutaneous

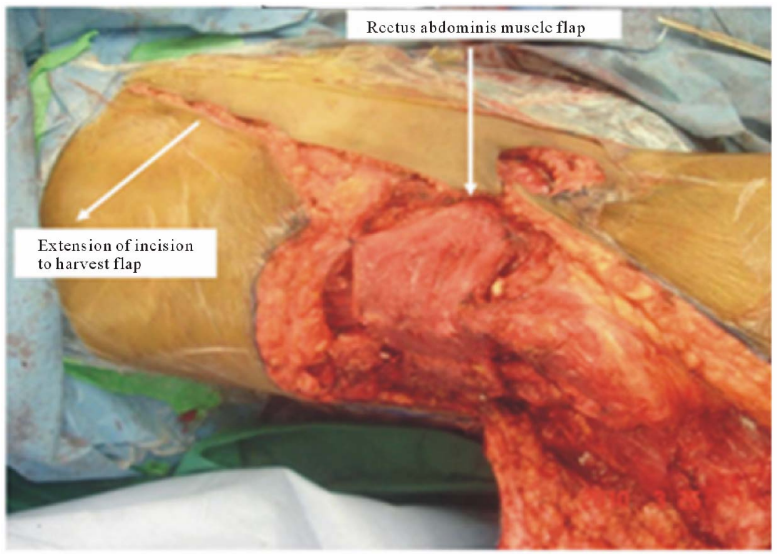

(b)

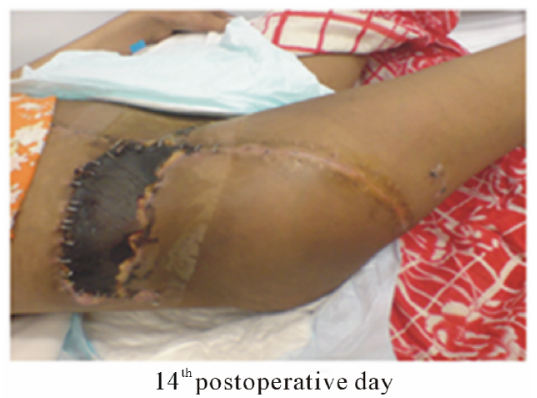

(d)

Figure 1. (a) Intraoperative photograph hemipelvectomy showing thin flap and nerves; (b) Nerves covered with rectus abdominis muscle flap; (c) Early postoperative photograph showing epidermal necrosis; (d) Full thickness necrosis over rectus abdominis musle cushion. 
component is harvested over main vessels from the amputated limb and is anastomosed with recipient vessel. We have used same type of reconstruction in two patients, one with forequarter amputation and another with external hemipelvectomy. Both patients had good take of flap in the recipient region.

Seven patients had soft tissue sarcomas for which all of them underwent wide margin excision followed by reconstruction with pedicled flaps in all except one in whom free lattissimus dorsi flap was done. Two patients among this group had malignant melanoma of upper and lower limb each. First patient was 65 years of age with heel tumor, cardiac arrythmias and ejection fraction of $25 \%$. Flap failure was a concern due to age and circulatory compromise but fortunately flap survived. In this case Breslow thickness of $13 \mathrm{~mm}$ was noted and a closest positive margin was $0.5 \mathrm{~cm}$ away. Recurrence noted in the form of a nodule away from the surgical site after two years. Excision of lesion with repeat biopsy was consistent with malignant melanoma. It is evident from the literature that melanoma of lower limb carries poor prognosis than upper limb disease [13]. In second patient with melanoma of wrist which was excised, axillary lymph node clearance was done. Initial wound healing and recovery was satisfactory. Resected mass was a margin positive at final histopathology with no nodal involvement. Re excision was offered which was denied. Disease recurred at the surgical site in the form of small lesion after one and half year and thus more radical procedure in the form of forearm amputation was done.

Vascularized fibula was done in six patients; five of them had osteogenic sarcoma and remaining one had pleomorphic sarcoma of forearm for which osteocutaneous free fibular flap was done to reconstruct the radius. Four patients had onlay grafting of vascularized fibula to augment biological reconstruction with autograft and autoclaved bone. One patient had it done for intercalary defect reconstruction. Viability of flap was checked with bone scan after 2 weeks. Tracer uptake by fibula was evident in all 6 cases. Union is noticed in all cases. Three out of five patients with lower limb reconstruction are ambulating full weight bearing without support, where as remaining two are still using walking aid (Figure 2).

Overall functional outcome was assessed using Musculoskeletal tumor society (MSTS) score. In our study mean score was 73.5\% (Table 3). Reduced functional

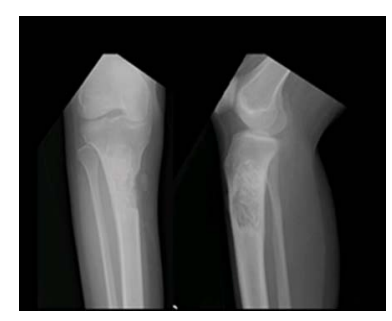

(a)

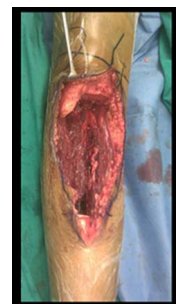

(d)

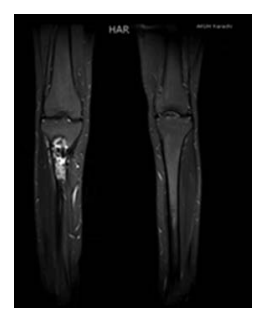

(b)

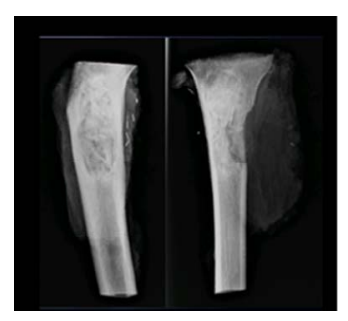

(c)

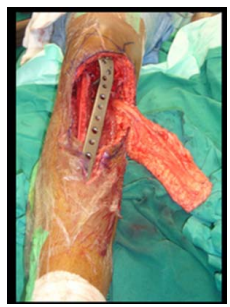

(e)

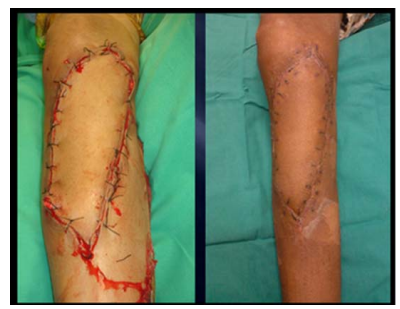

(f)

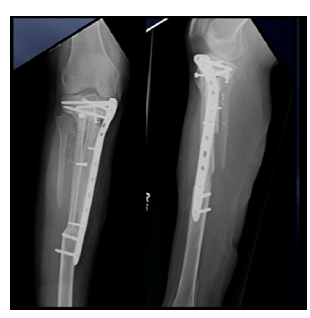

(g)

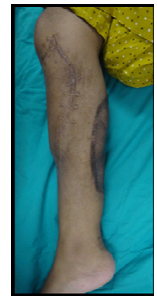

(h)

Figure 2. (a) and (b) Properative radiograph and MRI showing proximal tibia lesion; (c) Specimen radiograph; (d) Intraoperative picture showing remaining proximal tibia with tuberosity and sural pedical; (e) Biologic reconstruction with vascularized fibula and sural flap; (f) Post operative and 2 weeks post operative picture; (g) Thirty months follow up radiograph; (h) Thiry months follow up clinical photograph. 
Table 3. Comparision of our study and that of the other studies.

\begin{tabular}{ccc}
\hline Study & $\begin{array}{c}\text { Number of } \\
\text { patients }\end{array}$ & MSTS score (\%) \\
\hline Niimi R. et al. [14] & 63 & 81 \\
Li J. et al. [15] & 4 & 91.7 \\
Payne C. E. et al. [16] & 113 & 87 \\
Funovics P. T. et al. [17] & 28 & 85 \\
Haroon et al. & 24 & 73.5 \\
\hline
\end{tabular}

score compared to international literature is possibly due to cases with diverse age groups, with different pathologies. So far no study has been published from our part of the world describing their progress in musculoskeltaloncology. This may well be related to paucity of resources as well as specialized centers in the same field in our region.

\section{Conclusion}

Large soft tissue defects are the usual endpoint of wide surgical resections and coverage of those defects is essential. To overcome the fear of inadequate margins, one must not be afraid of the resultant wound size. It is imperative to have multidisciplinary team approach for the treatment of musculoskeletal tumor, especially when aiming for limb salvage with resultant functional limb. Development of sarcoma service demands high quality centers equipped with trained staff and resources for the management of cases and postoperative rehabilitation.

\section{REFERENCES}

[1] S. A. Rosenberg, J. Tepper, E. Glatstein, et al., "The Treatment of Soft-Tissue Sarcomas of the Extremities: Prospective Randomized Evaluations of 1) Limb-Sparing Surgery plus Radiation Therapy Compared with Amputation and 2) the Role of Adjuvant Chemotherapy," Annals of Surgery, Vol. 196, No. 3, 1982, pp. 305-315. http://dx.doi.org/10.1097/00000658-198209000-00009

[2] J. C. Yang, A. E. Chang, A. R. Baker, et al., "Randomized Prospective Study of the Benefit of Adjuvant Radiotherapy in the Treatment of Soft Tissue Sarcomas of the Extremity," Journal of Clinical Oncology, Vol. 16, No. 1, 1998, pp. 197-203.

[3] M. A. Clark, C. Fisher, I. Judson and J. M. Thomas, "Soft-Tissue Sarcomas in Adults," New England Journal of Medicine, Vol. 353, 2005, pp. 701-711. http://dx.doi.org/10.1056/NEJMra041866

[4] M. A. Clark and J. M. Thomas, "Amputation for SoftTissue Sarcoma,” Lancet Oncology, Vol. 4, No. 6, 2003, pp. 335-342. http://dx.doi.org/10.1016/S1470-2045(03)01113-6
[5] M. A. Clark and J. M. Thomas, "Major Amputation for Soft-Tissue Sarcoma,” British Journal of Surgery, Vol. 90, No. 1, 2003, pp. 102-107. http://dx.doi.org/10.1002/bjs.4004

[6] J. M. Serletti, A. J. Carras, R. J. O’Keefe and R. N. Rosier, "Functional Outcome after Soft-Tissue Reconstruction for Limb Salvage after Sarcoma Surgery,” Plastic and Reconstructive Surgery, Vol. 102, No. 5, 1998, pp. 1576-1583. http://dx.doi.org/10.1097/00006534-199810000-00036

[7] I. A. Kramers-de Quervain, J. M. Läuffer, K. Käch, O. Trentz and E. Stüssi, "Functional Donor-Site Morbidity during Level and Uphill Gait after a Gastrocnemius or Soleus Muscle-Flap Procedure,” The Journal of Bone and Joint Surgery (American Volume), Vol. 83, No. A2, 2001, pp. 239-246.

[8] T. Liu, Q. Zhang, X. Zhang, Z. Li, Y. Shen, X. Guo and L. Ling, "Medial Head Gastrocnemius Muscle Flap in the Limb-Salvage Operation for Proximal Tibial Osteosarcoma," Journal of Central South University. Medical Sciences, Vol. 37, No. 12, 2012, pp. 1250-1254.

[9] S. V. Deo, K. S. Nootan, B. Niranjan and K. Dinesh, "Vertical Rectus Abdominis Myocutaneous Flap Cover for Lower Abdomen, Chest Wall, Groin and Thigh Defects Following Resection of Malignant Tumours,” Indian Journal of Cancer, Vol. 38, No. 1, 2001, pp. 33-37.

[10] B. S. Glatt, J. J. Disa, B. J. Mehrara, A. L. Pusic, P. Boland and P. G. Cordeiro, "Reconstruction of Extensive Partial or Total Sacrectomy Defects with Transabdominal Vertical Rectus Abdomnis Myocutaneous Flap,” Annals of Plastic Surgery, Vol. 56, No. 5, 2006, pp. 526-530. http://dx.doi.org/10.1097/01.sap.0000205772.15061.39

[11] T. Morii, M. Susa, R. Nakayama, K. Kishi, H. Morioka and H. Yabe, "Reconstruction Modality Based on the Spare Part Concept for Massive Soft Tissue Defects Following Oncological Hemipelvectomy," Journal of Orthopaedic Science, Vol. 14, No. 2, 2009, pp. 192-197. http://dx.doi.org/10.1007/s00776-008-1316-5

[12] M. V. Küntscher, D. Erdmann, H. H. Homann, H. U. Steinau, S. L. Levin and G. Germann, "The Concept of Fillet Flaps: Classification, Indications, and Analysis of Their Clinical Value," Plastic and Reconstructive Surgery, Vol. 108, No. 4, 2001, pp. 885-896. http://dx.doi.org/10.1097/00006534-200109150-00011

[13] S. M. Walsh, S. G. Fisher and R. A. Sage, "Survival of Patients with Primary Pedal Melanoma,” Journal of Foot and Ankle Surgery, Vol. 42, No. 4, 2003, pp. 193-198. http://dx.doi.org/10.1016/S1067-2516(03)70028-3

[14] R. Niimi, A. Matsumine, T. Hamaguchi, T. Nakamura, A. Uchida and A. Sudo, "Prosthetic Limb Salvage Surgery for Bone and Soft Tissue Tumors around the Knee,” Oncology Reports, Vol. 28, No. 6, 2012, pp. 1984-1990.

[15] J. Li, Z. Wang, Z. Guo, M. Yang, G. Chen and G. Pei, "Composite Biological Reconstruction Following Total Calcanectomy of Primary Calcaneal Tumors," Journal of Surgical Oncology, Vol. 105, No. 7, 2012, pp. 673-678. http://dx.doi.org/10.1002/jso.23022

[16] C. E. Payne, S. O. Hofer, T. Zhong, A. C. Griffin, P. C. 
Ferguson and J. S. Wunder, "Functional Outcome Following upper Limb Soft Tissue Sarcoma Resection with Flap Reconstruction,” Journal of Plastic, Reconstructive \& Aesthetic Surgery, Vol. 66, No. 5, 2013, pp. 601-607. http://dx.doi.org/10.1016/j.bjps.2013.01.034
[17] P. T. Funovics, F. Bucher, C. D. Toma, R. I. Kotz and M. Dominkus, "Treatment and Outcome of Parosteal Osteosarcoma: Biological versus Endoprosthetic Reconstruction,” Journal of Surgical Oncology, Vol. 103, No. 8, 2011, pp. 782-789. http://dx.doi.org/10.1002/jso.21859 